\title{
A dilemma in management of a depression episode in a patient with hypothyroidism
}

\begin{abstract}
Background: The link between hypothyroidism and depression is a major concern in Endocrinology and Psychiatry.

Several animal studies have been conducted which shows that thyroid hormones influences serotonergic and noradrenergic neurotransmission which plays a key role in the pathogenesis of depression. In the studies, T3 have been administered to rats which resulted in an increase in serotonin levels in the cerebral cortex. Studies on rats have also shown a decrease in serotonin synthesis in the brain with hypothyroidism.
\end{abstract}

Objectives: A Management Dilemma in hypothyroid patients with a depressive episode. Whether to discontinue or continue anti-depressant when the patient reaches euthyroid state and becomes asymptomatic.

Method: A case study was done on a patient with hypothyroidism in a depression episode.

A 49 years old male came to the clinic complaining from Low mood since 3 months. The low mood was associated with lack of appetite, fatigue, decreased concentration, feeling of hopelessness and worthlessness, insomnia, loss of pleasure in pleasurable activities (anhedonia). Patient did not have suicidal ideations or intention. Patient also feels always anxious and has anticipation anxiety. No Psychotic symptoms or any other psychiatric manifestation. Past psychiatric history showed no previous depression episodes or any other psychiatric illness. Past medical history, patient suffers from Diabetes mellitus on oral hypoglycemic agents since 5 years. Family history revealed no depression or any other psychiatric illness that runs in the family.

Discussion: The dilemma is whether to stop the course of antidepressant in hypothyroid patients when the patient reaches a euthyroid state.

An association between thyroid function and depression in subjects on thyroxine was first shown by Saravanan et al.1 who found a clear association between higher TSH and lower free T4 and poorer psychological well-being in 697 subjects on thyroxine from the UK. This persisted when only subjects with a TSH within the reference range (indicating adequate replacement) were included. Several studies have been conducted around the world which proved the same association.

Conclusion: Thyroid hormones play a vital role in cognition and mood. Currently the correlation between thyroid function and psychiatric illness is an area of concern for further research. Hypothyroidism can lead to generalized anxiety disorder, depressive episode and disturbance in memory as proven by multiple studies. Patients who have a comorbidity of hypothyroidism and depression must be treated by an endocrinologist and a liaison psychiatrist to optimise management efficiency. Early recognition of hypothyroidism and management will help reduce psychiatric manifestations and hence improve overall quality of life before a person completes the criteria for depression or anxiety.
Volume 6 Issue 2 - 2018

\author{
Khalid Al Jalahma, 'Yahya Zaman, ${ }^{2}$ Waiel Al \\ $\mathrm{Ani}^{3}$ \\ 'Psychiatric Resident, Bahrain \\ ${ }^{2}$ Consultant Endocrinologist, Bahrain \\ ${ }^{3}$ Consultant Psychiatrist, Bahrain
}

Correspondence: Khalid Al Jalahma, Psychiatric Resident, Bahrain,Email dr_khalid89@hotmail.com

Received: January 15, 2018 | Published: March 12, 2018

\section{Introduction}

The link between hypothyroidism and depression is a major concern in Endocrinology and Psychiatry. Impairment in the secretion of Thyroxine which is the thyroid hormone is what leads to hypothyroidism. Most patients suffering from hypothyroidism are asymptomatic and the patients who are symptomatic usually have none specific symptoms. Those symptoms include Low mood, malaise and lethargy. There are several other symptoms including cold intolerance, weight gain, dry skin, constipation and cold intolerance.
Several animal studies have been conducted which shows that thyroid hormones influences serotonergic and noradrenergic neurotransmission which plays a key role in the pathogenesis of depression. In the studies, T3 have been administered to rats which resulted in an increase in serotonin levels in the cerebral cortex. Studies on rats have also shown a decrease in serotonin synthesis in the brain with hypothyroidism. ${ }^{2}$

The following is a short comparison of the symptoms of hypothyroidism, symptoms of depression and symptoms that are shared between the 2 disorders. 


\begin{tabular}{ll}
\hline & $\begin{array}{l}\text { Cold intolerance Hyperlipidemia Brittle } \\
\text { and dry hair loss Dry and thickened skin } \\
\text { Hypothyroidism } \\
\text { only }\end{array}$ \\
$\begin{array}{l}\text { Bradycardia Delayed re exes Myxedema } \\
\text { Decreased basal metabolic rate Anaemia } \\
\text { Menorrhagia Goiter }\end{array}$ \\
Depression only & $\begin{array}{l}\text { Weight loss, Sleep disturbance and Decreased } \\
\text { appetite }\end{array}$ \\
& $\begin{array}{l}\text { Depressed mood,Apathy, Emotional lability, } \\
\text { Decreased appetite Increased sleep, Weight } \\
\text { gain, Fatigue, Poor concentration, Mental } \\
\text { slowing and Diminished libido }\end{array}$ \\
\hline
\end{tabular}

\section{Case summary}

\section{History}

49 years old male came to the clinic complaining from Low mood since 3 months. The low mood was associated with lack of appetite, fatigue, decreased concentration, feeling of hopelessness and worthlessness, insomnia, loss of pleasure in pleasurable activities (anhedonia). Patient did not have suicidal ideations or intention. Patient also feels always anxious and has anticipation anxiety. No Psychotic symptoms or any other psychiatric manifestation. Past psychiatric history showed no previous depression episodes or any other psychiatric illness. Past medical history, patient suffers from Diabetes mellitus on oral hypoglycaemic agents since 5 years. Family history revealed no depression or any other psychiatric illness that runs in the family. Patient went through divorce 17 years ago; currently he is single with no children. His childhood history was smooth, no history of abuse. Patient achieved bachelor's degree in accounting and currently works as an accountant in a bank.

Patient was assessed using Beck's depression inventory and scored 24 which is considered moderate severity depression. Patient was also assessed using Hamilton anxiety rating scale and scored 22 which are considered anxiety of mild to moderate in severity.

\section{Mental state examination}

\section{Appearance}

Psychomotor retardation with reduced spontaneous movements, untidy hair and facial grooming, depressed facial expression with down turned corners of mouth, tearful.

\section{Speech}

Reduced rate, volume and tone. Coherent speech.

\section{Mood}

Low mood, low appetite and disturbed sleep

\section{Thoughts}

Depressive content including sense of hopelessness, worthlessness and helplessness. No suicidal ideations, no delusions.

\section{Perception and cognition}

No hallucinations

Impaired concentration

\section{Insight}

Preserved

\section{Investigations}

Lab investigations was requested for this patient including complete blood count, urea and electrolytes, Renal function test, Liver function test, Vitamin B12, Vitamin D and Thyroid function test. Patient was not started on any course of medications yet; he was given a follow up appointment after 1 week.

Upon follow up, all of his investigations were normal except his TFTs results which showed TSH 12.4 and FT4 13.2, FT3 5.7

Patient was started on a course of anti-depressant Duloxetine 30 $\mathrm{mg}$ pood (Anti-depressant from SNRI's class) and lorazepam $1 \mathrm{mg}$ poprn which is a benzodiazepine that would help him sleep better and control his anxiety. Patient was also referred to an endocrinologist and given 2 weeks follow up appointment. Patient was started on Thyroxine $0.1 \mathrm{mg}$ pood by the endocrinologist. After 2 months, patient reached euthyroid state and his anxiety and depression symptoms also improved. The course of anti-depressant was stopped, however patient had a relapse of a depressive episode after 1 month despite being treated for his Hypothyroidism.

\section{Discussion}

The dilemma is whether to stop the course of antidepressant in hypothyroid patients when the patient reaches a euthyroid state.

An association between thyroid function and depression in subjects on thyroxine was first shown by Saravanan et al. ${ }^{1}$ who found a clear association between higher TSH and lower free T4 and poorer psychological well-being in 697 subjects on thyroxine from the UK. This persisted when only subjects with a TSH within the reference range (indicating adequate replacement) were included. Several studies have been conducted around the world which proved the same association.

Thyroid hormones replacement therapy is very important as an adjuvant to antidepressant therapy in improving the mood. As mentioned earlier in animal studies it has shown that $\mathrm{T} 3$ have been playing a role in serotonin levels in the brain. Antidepressant has also been shown to increase deiodinase action which leads to increase in thyroid hormone concentration hence improving Thyroid hormone level regulation. ${ }^{3}$ Altshuler et al found an accelerated response to antidepressants with the addition of thyroid hormones, particularly in women, whilst Aronson et al found that the addition of T3 in subjects with resistant depression increased the response rate by $23 \%{ }^{4}$

Almost all psychiatrists request a thyroid function test as part of the workup before starting anti-depressant. This is because even mild cases of hypothyroidism might lead to depression.

Hypothyroidism usually leads to a mixed depression and anxiety state. Psychiatric manifestations are usually the first symptoms that appear in patients with hypothyroidism. Initially it starts with anxiety, memory lapses and mental slowing and finally may lead to completing the criteria of generalized anxiety disorder and/or Depressive episode.

Hypothyroidism affects cognitive functions in multiple dimensions. It can lead to poor learning, reduced efficiency in executive functions and slowed speed on information processing. Very severe hypothyroidism can lead to melancholic depression and pseudo dementia, hence leading to a reduction in quality of life.

Research suggests that Psychiatric symptoms improve when patient gets treated for his hypothyroidism and reach euthyroid state, 
however it does not completely resolve unless patient continues his antidepressant course. ${ }^{5}$

Thyroid hormones have also been effective in patients with treatment resistant depression and Thyroid function test is a good predictor for the risk of relapse in hypothyroid patients who suffer from depression or bipolar.

Thyroid hormones affect our Central and Peripheral nervous systems through our entire lives. It is involved in regulating myelination, differentiation of neuronal and glial cells, and neuronal viability and function. Thyroid hormones also stimulate the development of neuronal processes such as axons and dendrites and it increase the rate of neuronal proliferation. ${ }^{6}$

Thyroid hormone effect on serotonin is explained by desensitization of 5-HT1A auto receptors at the site of raphe nuclei which results in an increased release of serotonin from raphe neurons. ${ }^{7-20}$

\section{Conclusion}

Thyroid hormones play a vital role in cognition and mood. Currently the correlation between thyroid function and psychiatric illness is an area of concern for further research. Hypothyroidism can lead to generalized anxiety disorder, depressive episode and disturbance in memory as proven by multiple studies. Patients who have a comorbidity of hypothyroidism and depression must be treated by an endocrinologist and a liaison psychiatrist to optimise management efficiency. Early recognition of hypothyroidism and management will help reduce psychiatric manifestations and hence improve overall quality of life before a person completes the criteria for depression or anxiety. If a patient has hypothyroidism and he completes the criteria of a depressive episode, he must complete the full course of anti-depressant even if his depression symptoms resolved when he reaches euthyroid state. This is to avoid a relapse of a depressive episode.

Endocrinologists who have patients that are on treatment and achieved euthyroid state but still having anxiety or depression symptoms must be referred to a psychiatrist for a proper mental state examination and further management.

Psychiatrists should always screen for thyroid function prior to starting the course of antidepressant. Patients suffering from anxiety or depression and resistant to medications or requiring higher doses also need to be screened for thyroid function status.

\section{Acknowledgement}

None.

\section{Conflict of Interest}

None.

\section{References}

1. Saravanan P, Visser TJ, Dayan CM. Psychological well-being correlates with free thy- roxine but not free 3,5,3'-triiodothyronine levels in patients on thyroid hormone re- placement. J Clin Endocrinol Metab. 2006;91(9):3389-3393.
2. Kirkegaard C, Faber J. The role of thyroid hormones in depression. Eur J Endocrinol. 1998;138(1):1-9.

3. Heuer H. The importance of thyroid hormone transporters for brain development and function. Best Pract Res Clin Endocrinol Metab. 2007;21(2):265-276.

4. Altshuler LL, Bauer M, Frye MA, et al. Does thyroid supplementation accelerate tri-cyclic antidepressant response? A review and meta-analysis of the literature. Am J Psychiatry. 2001;158(10):1617-1622.

5. Jorde R, Waterloo K, Storhaug H, et al. Neuropsychologi- cal function and symptoms in subjects with subclinical hypothyroidism and the effect of thyroxine treatment. J Clin Endocrinol Metab. 2006;91(1):145-153.

6. Bauer M, Heinz A, Whybrow PC. Thyroid hormones, serotonin and mood: Of synergy and significance in the adult brain. Mol Psychiatry. 2002;7(2):140-156.

7. Bernal J. Thyroid hormones and brain development. Eur J Endocrinol. Vitam Horm. 2005;71:95-122.

8. Schwartz CE, May MM, Carpenter NJ, et al. Allan-Herndon-Dudley syndrome and the mono-carboxylate transporter 8 (MCT8) gene. Am J Hum Genet. 2005;77(1):41-53.

9. Bono G, Fancellu R, Blandini F, et al. Cognitive and affective status in mild hypothyroidism and interactions with L-thyroxine treatment. Acta Neurol Scand. 2004;110(1):59-66.

10. Tremont G, Stern RA, Westervelt HJ, et al. Neurobehavioral functioning in thyroid disorders. Med Health R. 2003;86(10):318-22.

11. Atterwill CK, Bunn SJ, Atkinson DJ, et al. Effects of thyroid status on presynaptic alpha 2-adrenoceptor function and beta-adrenoceptor binding in the rat brain. J Neural Transm. 1984;59(1):43-55.

12. Hall RC, Stickney S, Beresford TP. Endocrine disease and behaviour Integr Psychiatry. 1986;4:122-135.

13. Hamilton M. The assessment of anxiety states by rating. $\mathrm{Br} \mathrm{J} \mathrm{Med}$ Psychol. 1959;32(1):50-55.

14. Brown BT, Graham PL, Bonello R, et al. A biopsychosocial approach to primary hypothyroidism: Treatment and harms data from a randomized controlled trial. Chiropr Man Therap. 2015;23:24.

15. Haggerty JJ, Garbutt JC, Evans DL, et al. Subclinical hypothyroidism A review of neuropsychiatric aspects. Int $J$ Psychiatry Med. 1990;20(2):193-208.

16. Escobar-Morreale HF, Obregon MJ, Escobar del Rey F, et al. Replacement therapy for hypothyroidism with thyroxine alone does not ensure euthyroidism in all tissues, as studied in thyroidectomized rats. $J$ Clin Invest. 1995;96(6):2828-2838.

17. Parle JV, Franklyn JA, Cross KW, et al. Thyroxine prescription in the community: serum thyroid stimulating hormone level assays as an indicator of under- treatment or overtreatment. Br J Gen Pract. 1993;43(368):107-109.

18. Jaeschke R, Guyatt G, Gerstein H, et al. Does treatment with L-thyroxine influence health status in middle-aged and older adults with subclinical hypothyroidism? J Gen Intern Med.1996;11(12):744-749.

19. Samuels MH, Schuff KG, Carlson NE, et al. Health status, psychological symptoms, mood, and cognition in L-thyrox- ine-treated hypothyroid subjects. Thyroid. 2007;17(3):249-258.

20. LaFranchi S. Congenital hypothyroidism: eti-ologies, diagnosis, and management. Thyroid. 1999;9(7):735-740. 